\title{
Country Evaluation of Islamic Economic Criteria: An Application Using Entropy Weight and TOPSIS Methodologies
}

\author{
Mayor-Vitoria, F. ${ }^{1}$, García-Bernabeu, A. ${ }^{2}$, Nor Aida Abdul Rahman ${ }^{3}$, \\ Mohammad Fakhrulnizam Mohammad ${ }^{4}$ \\ ${ }^{1}$ UniversitatPolitècnica de València, Spain \\ fermavi[at]upv.es \\ ${ }^{2}$ Universitat Politècnica de València, Spain \\ ${ }^{3}$ Universiti Kuala Lumpur (MIAT Campus), Malaysia \\ ${ }^{4}$ Universiti Kuala Lumpur (MIAT Campus), Malaysia
}

\begin{abstract}
While Islamic economy is growing in all sectors, Halal compliance is carried out by more countries worldwide. The purpose of this paper is toestablish a methodology based on MCDM methods in order to create an accurate ranking of countries according to the basis of the Halal standards, which can be used by public and private institutions. Although Islamic economy is evaluated globally by different institutions every year, those reports are based on indicators which are equally weighted. In this paper, weights of the six Islamic economy criteria are determined by the EWM (Entropy Weight Method) and theTechnique for Order Preference by Similarity to Ideal Solution (TOPSIS) has been used to create a ranking of countries in terms of the Halalprinciples.
\end{abstract}

Keywords: Halal, MCDM, TOPSIS, Entropy, Islamic economy

\section{Introduction}

Islamic economy and Muslim consumers play a critical role in the global economy. In this context, several countries around the world are adapting their economies to the Halal principles in order to attract new Muslim consumers from all around the world [1]. According to [2], it is estimated that Muslims spent US\$2.2 trillion in 2018 across the food, pharmaceutical and lifestyle sectors which are impacted by Islamic faith inspired ethical consumption needs. This spending reflects a healthy $5.2 \%$ year-on-year growth and it is forecasted to reach US $\$ 3.2$ trillion by 2024 .

In this paper, evaluation criteria system of global Islamic economy is established on the basis of the Halal principles. Weights of criteria are determined by the Entropy Weight Method in order to weight the criteria according to the registered information. Those criteria of the Islamic economy in seventy countries are applied using TOPSIS method, which was firstly introduced by [3] in 1981.

Furthermore, results are compared with other evaluation methodologies and the case study reports that the method is clear and the results are reliable. So the methodology could be promoted in several fields for evaluating and ranking.

This study is focused on the measurement of the strengths of seventy countries in order to analyze their ecosystems in terms of the Islamic economy. Analyzing the Islamic economy is important in order to have a comprehensive picture of those countries in order to determinate which ones are better positioned to face this multi-trillion global opportunity.
Although this has been measured since 2013when the report was firstly published [4], in this research a more accurate methodology is applied and the results show some differences in the terms of the final ranking of the countries involved because the methodology is not simply based on equally weighted methods.

As in previous reports, results show that leading countries such as UAE and Malaysia are maintaining the first positions but in different order. When talking about fast growing countries such as, Turkey or Indonesia, results are also different in terms of final ranking positions.

\section{Materials and Methods}

\subsection{Criteria's selection}

On the basis of [2] and as it is shown in table 1.six indicators have been identified as criteria to be used in this research. These criteria focus on the four pillars of Islamic economy, that is to say, consumers, business, governments and investors.

Table 1: List of criteria

\begin{tabular}{|l|l|}
\hline Criteria & Symbol \\
\hline IslamicFinance & $\mathrm{C}_{1}$ \\
\hline Halal Food & $\mathrm{C}_{2}$ \\
\hline Halal Travel & $\mathrm{C}_{3}$ \\
\hline ModestFashion & $\mathrm{C}_{4}$ \\
\hline Halal Media and Recreation & $\mathrm{C}_{5}$ \\
\hline Halal Pharmaceuticals and Cosmetics & $\mathrm{C}_{6}$ \\
\hline
\end{tabular}




\section{International Journal of Science and Research (IJSR) \\ ISSN: 2319-7064 \\ SJIF (2019): 7.583}

\subsection{Weights of the criteria}

Determining weights of criteria can be a done using subjective methods such as the Analytic Hierarchy Process Method (AHP) introduced by[5] in the 70s,but they could involve deviations because of subjective factors.In this case,Entropy Weight Method (EWM) is applied as an objective fixed weights method in order to determine the weights of the criteria based on the amount of inherent information, so the man-made disturbances are eliminated. Entropy weight method has been applied as follows.

\subsubsection{Standardization of criteria}

Supposing there are $m$ countries and $n$ criteria, $x_{i j}$ is the $j^{\text {th }}$ criteria value in the $i^{\text {th }}$ country. In order to eliminate the influence of criteria on incommensurability,it is necessary to standardize criteria using the equations of relative optimum membership degree. For the benefit criteria, the attribute value of the $j^{\text {th }}$ criterion in the $i^{\text {th }}$ country can be transformed by (1)

$$
r_{i j}^{\prime}=\frac{x_{i j}}{\max _{j} x_{i j}},(i=1, \ldots, m ; j=1, \ldots, n)
$$

For the cost criteria, the attribute value of the $j^{\text {th }}$ criterion in the $i^{\text {th }}$ country can be transformed by (2)

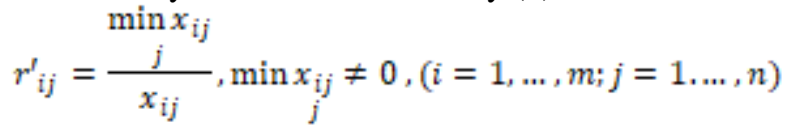

\subsubsection{Calculation of the index's entropy}

Entropy of the $j^{\text {th }}$ index is determined by (3) and (4)

$$
H_{j}=-\frac{\sum_{i=1}^{m} f_{i j} \ln f_{i j}}{\ln m},(i=1, \ldots, m ; j=1 . \ldots, n)
$$

Where:

$$
f_{i j}=-\frac{r_{i j}^{\prime}}{\sum_{i=1}^{m} r_{i j}},(i=1, \ldots, m ; j=1, \ldots, n)
$$

2.2.3 Calculation of the criteria's entropy weight as in (5)

$$
w_{j}=\frac{1-H_{j}}{n-\sum_{j=1}^{n} H_{j}}, \sum_{j=1}^{n} w_{j}=1,(j=1 . \ldots, n)
$$

Therefore, the bigger the entropy weight is, the more important the criteria are. Therefore, as is shown in the case study, pharmaceutical and halal food are considered the most important criteria.

\subsection{Applying TOPSIS method}

Technique for Order Preference by Similarity to Ideal Solution (TOPSIS) has been used in several applications, like [6]in logistics or [7] for a warehouse's location selection problem, and the basic thought is to find the ideal positive and negative solution for decision making problems first.

Then, the main aim consists offinding a feasible solution and ranking a set of alternatives (countries) according to the closeness between the feasible solution and the ideal solution, which is made the nearest to the ideal solution and farthest from the negative ideal solution. The method has been used as follows.

\subsubsection{Structure of the decision matrix}

Criteria set is $C=\left(C_{1} C_{2} \ldots, C_{n}\right)$, the $j^{\text {th }}$ value index in the $i^{\text {th }}$ country is $x_{i j}$, then the decision matrix is $X=\left[x_{i j}\right] m \times n$.

\subsubsection{Normalization of the decision matrix}

In order to eliminate the influence of index dimension and its variation range on evaluation results, it isnecessary to normalize the original matrix to ensure that all the attributes are equivalent and the sameformat, then the normalized decision matrix is $R=\left[r_{i j}\right] m \times n$, which is calculated by (6)

$$
r_{i j}=\frac{x_{i j}}{\sqrt{\sum_{j=1}^{m} x_{i j}^{2}}},(i=1, \ldots, m ; j=1 . \ldots, n)
$$

\subsubsection{Determination of the weighted decision matrix}

The weighted decision matrix is determined by the normalized decision matrix multiplication with weights of indexes and shown by (7)

$$
v_{i j}=w_{i} r_{i j},(\mathrm{i}=1, \ldots, \mathrm{m} ; \mathrm{j}=1 . \ldots, \mathrm{n})
$$

\subsubsection{Determination of the ideal solution}

The ideal solution is composed of the optimal value of every attribute from the weighted decisionmatrix and shown by (8), and the negative ideal solution is composed of the worst value of every attributefrom the weighted decision matrix and shown by (9)

$$
\begin{aligned}
& V^{+}=\left(V_{1}{ }^{+}, V_{2}{ }^{+}, \ldots, V_{m}{ }^{+}\right) \\
& V^{-}=\left(V_{1}{ }^{-}, V_{2}{ }^{-}, \ldots, V_{m}{ }^{-}\right)
\end{aligned}
$$

Wherein, the ideal value and negative ideal value are determined by (10) and (11)

$$
\begin{gathered}
V_{j}^{+}=\left\{\begin{array}{c}
\max v_{i j}, \text { the benifit criteria } \\
\min v_{i j}, \text { the cost criteria }
\end{array}\right. \\
V_{j}^{-}=\left\{\begin{array}{c}
\max v_{i j}, \text { the cost criteria } \\
\min v_{i j}, \text { the benifit criteria }
\end{array}\right.
\end{gathered}
$$

\subsubsection{Calculation of the distance}

The distance of every feasible solution from the ideal solution and the negative ideal solution iscalculated respectively by (12) and (13)

$$
\begin{aligned}
& S_{i}^{+}=\sqrt{\sum_{j=1}^{n}\left(v_{i j}-v_{j}^{+}\right)^{2}},(\mathrm{i}=1, \ldots, \mathrm{m} ; \mathrm{j}=1, \ldots, \mathrm{n}) \\
& S_{i}{ }^{-}=\sqrt{\sum_{j=1}^{n}\left(v_{i j}-v_{j}^{-}\right)^{2}},(\mathrm{i}=1, \ldots, \mathrm{m} ; \mathrm{j}=1, \ldots, \mathrm{n})
\end{aligned}
$$

2.3.6 Calculation of the relative degree of approximation The relative degree of approximation is determined by (14)

$$
C_{i}=\frac{S_{i}^{-}}{\left(S_{i}^{+}-S_{i}^{-}\right)},\left(0 \leq C_{i} \leq 1 ; i=1.2 \ldots, \mathrm{m}\right)
$$

The evaluation object is ranked according to the value of the relative degree of approximation. The bigger the value is, the better the evaluation object is.

\section{Volume 10 Issue 2, February 2021 www.ijsr.net}


All the criteria above mentioned for seventy countries are evaluated according to surveyed data from the Global Islamic Economic Report 2019 and results are shown in table 2 .

Table 2: The surveyed data of evaluation criteria of the seventy countries

\begin{tabular}{|c|c|c|c|c|c|c|}
\hline & $\begin{array}{l}\text { Islamic } \\
\text { Finance }\end{array}$ & $\begin{array}{l}\text { Halal } \\
\text { Food }\end{array}$ & $\begin{array}{l}\text { Halal } \\
\text { Travel }\end{array}$ & $\begin{array}{l}\text { Modest } \\
\text { Fashion } \\
\end{array}$ & $\begin{array}{l}\text { Halal Media } \\
\text { \& Recreation }\end{array}$ & $\begin{array}{c}\text { Halal Pharmaceuticals } \\
\text { \& Cosmetics }\end{array}$ \\
\hline Afghanistan & 13.78 & 28.65 & 18.82 & 1.63 & 7.64 & 25.5 \\
\hline Albania & 0.73 & 14.73 & 34.55 & 18.73 & 25.03 & 14.9 \\
\hline Algeria & 2.24 & 38.64 & 22.42 & 11.37 & 16.21 & 40.63 \\
\hline Australia & 3.81 & 59.47 & 15.86 & 16.75 & 36.66 & 25.15 \\
\hline Azerbaijan & 1.81 & 42.45 & 40.53 & 8.72 & 33.53 & 44.43 \\
\hline Bahrain & 86.26 & 44.85 & 19.83 & 19.01 & 43.79 & 45.47 \\
\hline Bangladesh & 33.26 & 34.81 & 18.99 & 27.83 & 6.9 & 33.9 \\
\hline Benin & 0.22 & 23.85 & 3.05 & 16.41 & 4.59 & 28.16 \\
\hline Brazil & 0.02 & 62.82 & 3.39 & 10.29 & 23.26 & 14.61 \\
\hline Brunei & 43.22 & 57.99 & 26.06 & 11.65 & 37.16 & 57.26 \\
\hline Burkina Faso & 0.52 & 32.08 & 10.55 & 22.25 & 5.26 & 35.19 \\
\hline Cameroon & 1.6 & 24.44 & 22.27 & 17.26 & 9.43 & 23.86 \\
\hline Canada & 2.07 & 27.05 & 7.93 & 22.5 & 37.04 & 22.82 \\
\hline Chad & 0.21 & 13.39 & 10.4 & 18.8 & 1.89 & 16.49 \\
\hline China & 0.06 & 22.99 & 6.27 & 31.2 & 35.01 & 20.89 \\
\hline Djibouti & 10.64 & 18.16 & 16.84 & 17.36 & 4.95 & 12.43 \\
\hline Egypt & 14.61 & 37.5 & 30.91 & 15.34 & 17.19 & 50.24 \\
\hline France & 0.74 & 22.85 & 12.6 & 29.49 & 38.56 & 39.61 \\
\hline Gabon & 0.23 & 16.74 & 11.56 & 6.73 & 18.12 & 18.93 \\
\hline Gambia & 6.76 & 17.05 & 25.96 & 0 & 6.97 & 17.34 \\
\hline Germany & 0.19 & 19.78 & 8.27 & 26.33 & 38.61 & 27.14 \\
\hline Guinea & 0.46 & 26.07 & 12.27 & 10.15 & 26.92 & 31.01 \\
\hline Guinea-Bissau & 0.3 & 14.77 & 0 & 9.92 & 1.42 & 19.3 \\
\hline Guyana & 0.06 & 0.03 & 4.25 & 0 & 13.44 & 0.68 \\
\hline India & 3.01 & 44.2 & 3.79 & 27.09 & 12.81 & 41.28 \\
\hline Indonesia & 46.45 & 47.82 & 64.58 & 34.26 & 16.03 & 44.39 \\
\hline Iran & 37.4 & 36.03 & 19.02 & 10.98 & 21.51 & 37.17 \\
\hline Iraq & 13.74 & 39.91 & 12.99 & 12.74 & 8 & 38.46 \\
\hline Italy & 0.5 & 18.48 & 14.9 & 30.92 & 30.39 & 19.87 \\
\hline Ivory Coast & 0.41 & 14.84 & 24.19 & 9.4 & 10.04 & 20.6 \\
\hline Jordan & 49.09 & 60.2 & 34.98 & 22.54 & 25.29 & 58.29 \\
\hline Kazakhstan & 12.11 & 32.21 & 14.4 & 11.29 & 30.17 & 32.38 \\
\hline Kenya & 15.31 & 25.53 & 7.27 & 27.18 & 13.71 & 28.45 \\
\hline Kuwait & 56.52 & 42.1 & 12.36 & 12.14 & 30.4 & 34.44 \\
\hline Kyrgyzstan & 5.03 & 15.01 & 31.28 & 7.11 & 16.08 & 19.81 \\
\hline Lebanon & 9.38 & 18.86 & 21.63 & 10.87 & 44.36 & 27 \\
\hline Libya & 5.17 & 25.33 & 19.05 & 4.13 & 7.64 & 25.04 \\
\hline Malaysia & 172.83 & 80.5 & 91.5 & 32.79 & 50.53 & 95.57 \\
\hline Mali & 0.25 & 34.19 & 19.9 & 24.41 & 4.19 & 37.53 \\
\hline Mauritania & 1.93 & 25.61 & 16.67 & 24.76 & 6.79 & 27.79 \\
\hline Morocco & 14.71 & 41.32 & 29.37 & 25.33 & 26.46 & 41.78 \\
\hline Mozambique & 0.22 & 26.25 & 24.87 & 7.64 & 6.61 & 27.53 \\
\hline Niger & 0.43 & 22.68 & 19.11 & 16.27 & 1.63 & 25.42 \\
\hline Nigeria & 26.21 & 7.78 & 21.1 & 12.33 & 10.54 & 12.12 \\
\hline Oman & 51.57 & 62.49 & 28.1 & 25.19 & 28.05 & 43.33 \\
\hline Pakistan & 52.98 & 57.86 & 15.04 & 22.11 & 9.34 & 58.12 \\
\hline Palestine & 22.35 & 21.79 & 17.91 & 15.77 & 23.07 & 21.5 \\
\hline Qatar & 54.68 & 49.39 & 27.17 & 11.59 & 62.92 & 35.08 \\
\hline Russia & 0.62 & 21.18 & 14.59 & 17.46 & 35.57 & 20.42 \\
\hline Saudi Arabia & 64.02 & 48.47 & 34.37 & 16.14 & 32.77 & 46.57 \\
\hline Senegal & 1.49 & 39.75 & 23.63 & 20.74 & 9.83 & 43.46 \\
\hline Sierra Leone & 0.3 & 8.83 & 1.91 & 18.87 & 4.44 & 13 \\
\hline Somalia & 3.45 & 25.24 & 0 & 4.13 & 0.71 & 8.33 \\
\hline South Africa & 24.45 & 41.33 & 9.27 & 22.4 & 21.85 & 35.38 \\
\hline Sri Lanka & 22.9 & 25.63 & 8.31 & 27.68 & 13.38 & 26.98 \\
\hline Sudan & 33.87 & 54.81 & 29.26 & 7.68 & 10.56 & 20.57 \\
\hline Suriname & 0.4 & 13.89 & 10.51 & 4.39 & 17.12 & 14.72 \\
\hline Syria & 6.83 & 7.92 & 8.68 & 10.61 & 14.7 & 2.42 \\
\hline
\end{tabular}

Volume 10 Issue 2, February 2021 www.ijsr.net

Licensed Under Creative Commons Attribution CC BY 
International Journal of Science and Research (IJSR)

ISSN: 2319-7064

SJIF (2019): 7.583

\begin{tabular}{|c|c|c|c|c|c|c|}
\hline Tajikistan & 4.29 & 38.26 & 4.99 & 8.24 & 7.92 & 44.43 \\
\hline Thailand & 4.51 & 40.91 & 42.14 & 26.81 & 20.84 & 43.56 \\
\hline Togo & 0.11 & 14.59 & 12.96 & 12.95 & 4.36 & 23.18 \\
\hline Tunisia & 10.01 & 37.72 & 40.63 & 14.12 & 19.7 & 39.08 \\
\hline Turkey & 20.69 & 44.46 & 70.92 & 31.93 & 24.98 & 40.63 \\
\hline Turkmenistan & 0.07 & 25.99 & 0 & 0 & 6.78 & 26.74 \\
\hline Uganda & 4.91 & 33.96 & 14.8 & 19.61 & 8.25 & 35.7 \\
\hline United Arab Emirates & 82.53 & 90.55 & 97.08 & 106 & 108.6 & 103.77 \\
\hline United Kingdom & 16.9 & 34.05 & 10.55 & 19.54 & 41.29 & 32.91 \\
\hline United States & 0.36 & 29.84 & 5.27 & 13.69 & 31.61 & 23.35 \\
\hline Uzbekistan & 0.65 & 16.75 & 1.12 & 0.89 & 17.81 & 16.72 \\
\hline Yemen & 6.62 & 34.34 & 2.66 & 15.74 & 9.27 & 38.55 \\
\hline
\end{tabular}

\subsection{Calculation of the entropy weight}

According to evaluation criteria which are the benefit indexes or the cost indexes, standardization of criteria is calculated by (1) (2) and shown as follows in table 3 .

Table 3: Normalized criteria

\begin{tabular}{|c|c|c|c|c|c|c|}
\hline & $\begin{array}{l}\text { Islamic } \\
\text { Finance } \\
\end{array}$ & Halal Food & Halal Travel & $\begin{array}{l}\text { Modest } \\
\text { Fashion } \\
\end{array}$ & $\begin{array}{c}\text { Halal Media \& } \\
\text { Recreation }\end{array}$ & $\begin{array}{c}\text { Halal Pharmaceuticals } \\
\text { \& Cosmetics }\end{array}$ \\
\hline Afghanistan & 0.0116183 & 0.01256573 & 0.01326835 & 0.00132716 & 0.005274495 & 0.011489799 \\
\hline Albania & 0.00061548 & 0.0064605 & 0.024358209 & 0.01525017 & 0.017280183 & 0.006713647 \\
\hline Algeria & 0.00188861 & 0.01694729 & 0.015806398 & 0.00925758 & 0.011191042 & 0.01830708 \\
\hline Australia & 0.00321232 & 0.02608322 & 0.011181511 & 0.01363803 & 0.02530929 & 0.011332096 \\
\hline Azerbaijan & 0.00152606 & 0.01861834 & 0.028574188 & 0.00709992 & 0.023148404 & 0.020019285 \\
\hline Bahrain & 0.07272819 & 0.01967097 & 0.013980413 & 0.01547815 & 0.030231691 & 0.020487888 \\
\hline Bangladesh & 0.02804243 & 0.01526748 & 0.013388202 & 0.02265949 & 0.004763614 & 0.015274674 \\
\hline Benin & 0.00018549 & 0.01046048 & 0.002150291 & 0.0133612 & 0.003168839 & 0.012688343 \\
\hline Brazil & $1.6863 \mathrm{E}-05$ & 0.02755251 & 0.002389995 & 0.00837823 & 0.016058213 & 0.006582979 \\
\hline Brunei & 0.03643998 & 0.0254341 & 0.018372646 & 0.00948556 & 0.025654479 & 0.025800231 \\
\hline Burkina Faso & 0.00043843 & 0.01407011 & 0.00743789 & 0.01811619 & 0.003631393 & 0.015855922 \\
\hline Cameroon & 0.001349 & 0.01071925 & 0.015700646 & 0.01405328 & 0.006510273 & 0.010750847 \\
\hline Canada & 0.00174527 & 0.01186398 & 0.005590755 & 0.01831975 & 0.025571634 & 0.010282244 \\
\hline Chad & 0.00017706 & 0.00587278 & 0.007332138 & 0.01530717 & 0.001304816 & 0.00743007 \\
\hline China & $5.0588 \mathrm{E}-05$ & 0.01008329 & 0.004420433 & 0.02540338 & 0.024170165 & 0.009412623 \\
\hline Djibouti & 0.00897088 & 0.00796488 & 0.011872424 & 0.0141347 & 0.003417375 & 0.005600714 \\
\hline Egypt & 0.0123181 & 0.0164473 & 0.02179196 & 0.01249 & 0.011867613 & 0.022637157 \\
\hline France & 0.00062391 & 0.01002189 & 0.008883167 & 0.02401108 & 0.02662101 & 0.017847488 \\
\hline Gabon & 0.00019392 & 0.00734207 & 0.008149954 & 0.00547964 & 0.012509665 & 0.008529486 \\
\hline Gambia & 0.00569954 & 0.00747804 & 0.018302145 & $8.1421 \mathrm{E}-07$ & 0.004811941 & 0.007813063 \\
\hline Germany & 0.00016019 & 0.0086754 & 0.00583046 & 0.02143817 & 0.026655529 & 0.012228751 \\
\hline Guinea & 0.00038784 & 0.01143416 & 0.008650513 & 0.00826424 & 0.018584999 & 0.013972497 \\
\hline Guinea-Bissau & 0.00025294 & 0.00647804 & $7.05013 \mathrm{E}-07$ & 0.00807697 & 0.000980338 & 0.008696201 \\
\hline Guyana & $5.0588 \mathrm{E}-05$ & $1.3158 \mathrm{E}-05$ & 0.002996306 & $8.1421 \mathrm{E}-07$ & 0.009278692 & 0.000306395 \\
\hline India & 0.00253781 & 0.01938588 & 0.002672 & 0.02205697 & 0.008843753 & 0.018599957 \\
\hline Indonesia & 0.03916328 & 0.02097359 & 0.045529758 & 0.02789487 & 0.011066773 & 0.020001262 \\
\hline Iran & 0.03153297 & 0.01580256 & 0.013409353 & 0.00894004 & 0.01485005 & 0.016748072 \\
\hline Iraq & 0.01158457 & 0.01750431 & 0.009158122 & 0.01037305 & 0.005523031 & 0.01732932 \\
\hline Italy & 0.00042156 & 0.00810523 & 0.010504698 & 0.0251754 & 0.020980614 & 0.008953032 \\
\hline Ivory Coast & 0.00034568 & 0.00650874 & 0.017054271 & 0.00765358 & 0.006931404 & 0.009281955 \\
\hline Jordan & 0.04138914 & 0.02640339 & 0.024661364 & 0.01835231 & 0.017459682 & 0.026264328 \\
\hline Kazakhstan & 0.01021028 & 0.01412713 & 0.010152191 & 0.00919244 & 0.020828731 & 0.014589792 \\
\hline Kenya & 0.01290828 & 0.01119732 & 0.005125447 & 0.02213025 & 0.009465094 & 0.012819011 \\
\hline Kuwait & 0.04765358 & 0.01846483 & 0.008713964 & 0.00988452 & 0.020987518 & 0.015517987 \\
\hline Kyrgyzstan & 0.00424093 & 0.0065833 & 0.022052815 & 0.00578904 & 0.011101292 & 0.008925997 \\
\hline Lebanon & 0.00790854 & 0.00827189 & 0.015249437 & 0.00885047 & 0.030625207 & 0.012165669 \\
\hline Libya & 0.00435897 & 0.0111096 & 0.013430503 & 0.00336269 & 0.005274495 & 0.011282532 \\
\hline Malaysia & 0.14571775 & 0.03530686 & 0.064508715 & 0.02669798 & 0.034884845 & 0.043061964 \\
\hline Mali & 0.00021078 & 0.01499555 & 0.014029764 & 0.01987489 & 0.002892688 & 0.01691028 \\
\hline Mauritania & 0.00162724 & 0.01123241 & 0.011752571 & 0.02015986 & 0.004687673 & 0.012521628 \\
\hline Morocco & 0.01240241 & 0.01812273 & 0.02070624 & 0.02062396 & 0.018267425 & 0.018825247 \\
\hline Mozambique & 0.00018549 & 0.01151311 & 0.01753368 & 0.00622057 & 0.004563404 & 0.012404477 \\
\hline Niger & 0.00036254 & 0.00994732 & 0.013472804 & 0.01324721 & 0.001125318 & 0.011453752 \\
\hline Nigeria & 0.02209838 & 0.00341227 & 0.01487578 & 0.01003922 & 0.007276593 & 0.005461034 \\
\hline Oman & 0.04348009 & 0.02740777 & 0.019810873 & 0.02050997 & 0.019365128 & 0.019523646 \\
\hline Pakistan & 0.0446689 & 0.02537708 & 0.0106034 & 0.0180022 & 0.006448139 & 0.02618773 \\
\hline
\end{tabular}

Volume 10 Issue 2, February 2021 www.ijsr.net

Licensed Under Creative Commons Attribution CC BY 
International Journal of Science and Research (IJSR)

ISSN: 2319-7064

SJIF (2019): 7.583

\begin{tabular}{|c|c|c|c|c|c|c|}
\hline Palestine & 0.0188439 & 0.00955698 & 0.012626788 & 0.01284011 & 0.015927041 & 0.009687477 \\
\hline Qatar & 0.04610222 & 0.02166219 & 0.019155211 & 0.0094367 & 0.043438639 & 0.015806359 \\
\hline Russia & 0.00052274 & 0.00928943 & 0.010286144 & 0.01421612 & 0.024556777 & 0.009200851 \\
\hline Saudi Arabia & 0.05397703 & 0.02125868 & 0.024231306 & 0.01314136 & 0.022623716 & 0.020983527 \\
\hline Senegal & 0.00125626 & 0.01743413 & 0.016659464 & 0.01688673 & 0.006786424 & 0.019582222 \\
\hline Sierra Leone & 0.00025294 & 0.00387279 & 0.001346575 & 0.01536416 & 0.003065282 & 0.005857545 \\
\hline Somalia & 0.00290879 & 0.01107013 & $7.05013 \mathrm{E}-07$ & 0.00336269 & 0.000490169 & 0.003753334 \\
\hline South Africa & 0.02061447 & 0.01812711 & 0.006535473 & 0.01823832 & 0.015084779 & 0.015941533 \\
\hline Sri Lanka & 0.01930762 & 0.01124118 & 0.00585866 & 0.02253736 & 0.009237269 & 0.012156658 \\
\hline Sudan & 0.02855673 & 0.02403937 & 0.020628689 & 0.00625314 & 0.007290401 & 0.009268438 \\
\hline Suriname & 0.00033725 & 0.00609208 & 0.00740969 & 0.00357439 & 0.011819286 & 0.006632543 \\
\hline Syria & 0.00575856 & 0.00347367 & 0.006119515 & 0.00863878 & 0.01014857 & 0.001090404 \\
\hline Tajikistan & 0.00361702 & 0.01678063 & 0.003518016 & 0.0067091 & 0.005467801 & 0.020019285 \\
\hline Thailand & 0.00380251 & 0.0179429 & 0.02970926 & 0.02182899 & 0.014387496 & 0.01962728 \\
\hline Togo & $9.2744 \mathrm{E}-05$ & 0.00639909 & 0.009136972 & 0.01054403 & 0.003010052 & 0.010444452 \\
\hline Tunisia & 0.00843971 & 0.01654379 & 0.02864469 & 0.01149666 & 0.013600464 & 0.01760868 \\
\hline Turkey & 0.01744431 & 0.01949991 & 0.049999542 & 0.02599775 & 0.017245664 & 0.01830708 \\
\hline Turkmenistan & $5.9019 \mathrm{E}-05$ & 0.01139907 & $7.05013 \mathrm{E}-07$ & $8.1421 \mathrm{E}-07$ & 0.004680769 & 0.012048518 \\
\hline Uganda & 0.00413976 & 0.01489467 & 0.010434197 & 0.01596668 & 0.005695626 & 0.016085718 \\
\hline UnitedArabEmirates & 0.06958333 & 0.03971474 & 0.068442689 & 0.08630636 & 0.074975146 & 0.046756723 \\
\hline UnitedKingdom & 0.01424886 & 0.01493415 & 0.00743789 & 0.01590968 & 0.028505744 & 0.014828599 \\
\hline UnitedStates & 0.00030353 & 0.01308766 & 0.00371542 & 0.01114655 & 0.021822876 & 0.010521051 \\
\hline Uzbekistan & 0.00054803 & 0.00734646 & 0.000789615 & 0.00072465 & 0.012295648 & 0.007533703 \\
\hline Yemen & 0.00558151 & 0.01506134 & 0.001875335 & 0.01281568 & 0.006399812 & 0.017369872 \\
\hline
\end{tabular}

Weighs of ten indexes are calculated by (3) (5) and shown in the table 4

Table 4: Weight of criteria

\begin{tabular}{|c|c|c|c|c|c|c|}
\hline & $\mathrm{C}_{1}$ & $\mathrm{C}_{2}$ & $\mathrm{C}_{3}$ & $\mathrm{C}_{4}$ & $\mathrm{C}_{5}$ & $\mathrm{C}_{6}$ \\
\hline Wei & 0.0845 & 0.1961 & 0.16749 & 0.1814 & 0.17395 & 0.19633 \\
ght & 5736 & 8964 & 4802 & 6325 & 8984 & 5968 \\
\hline
\end{tabular}

\subsection{Determination of evaluation rank}

The decision matrix of seventy countries decision-making and six evaluation criteria are established according to the data in the table 2. The normalized decision matrix is established by (6) and shown as follows in table 5 .

Table 5: Normalized matrix

\begin{tabular}{|c|c|c|c|c|c|c|}
\hline & $\begin{array}{l}\text { Islamic } \\
\text { Finance }\end{array}$ & Halal Food & Halal Travel & $\begin{array}{l}\text { Modest } \\
\text { Fashion }\end{array}$ & $\begin{array}{c}\text { Halal Media \& } \\
\text { Recreation }\end{array}$ & $\begin{array}{c}\text { Halal Pharmaceuticals } \\
\text { \& Cosmetics }\end{array}$ \\
\hline Afghanistan & 0.050390 & 0.093147 & 0.082034 & 0.008744 & 0.033944 & 0.084612 \\
\hline Albania & 0.002669 & 0.047890 & 0.150599 & 0.100480 & 0.111208 & 0.049440 \\
\hline Algeria & 0.008191 & 0.125626 & 0.097726 & 0.060996 & 0.072021 & 0.134816 \\
\hline Australia & 0.013932 & 0.193348 & 0.069132 & 0.089858 & 0.162879 & 0.083451 \\
\hline Azerbaijan & 0.006619 & 0.138013 & 0.176665 & 0.046780 & 0.148973 & 0.147425 \\
\hline Bahrain & 0.315432 & 0.145816 & 0.086436 & 0.101982 & 0.194558 & 0.150876 \\
\hline Bangladesh & 0.121624 & 0.113174 & 0.082775 & 0.149298 & 0.030657 & 0.112485 \\
\hline Benin & 0.000804 & 0.077541 & 0.013295 & 0.088034 & 0.020393 & 0.093439 \\
\hline Brazil & 0.000073 & 0.204240 & 0.014777 & 0.055202 & 0.103344 & 0.048478 \\
\hline Brunei & 0.158045 & 0.188537 & 0.113592 & 0.062498 & 0.165101 & 0.189996 \\
\hline Burkina Faso & 0.001902 & 0.104298 & 0.045986 & 0.119363 & 0.023370 & 0.116765 \\
\hline Cameroon & 0.005851 & 0.079459 & 0.097072 & 0.092594 & 0.041897 & 0.079171 \\
\hline Canada & 0.007569 & 0.087945 & 0.034566 & 0.120704 & 0.164568 & 0.075720 \\
\hline Chad & 0.000768 & 0.043533 & 0.045332 & 0.100855 & 0.008397 & 0.054716 \\
\hline China & 0.000219 & 0.074745 & 0.027330 & 0.167377 & 0.155548 & 0.069316 \\
\hline Djibouti & 0.038908 & 0.059042 & 0.073403 & 0.093130 & 0.021993 & 0.041244 \\
\hline Egypt & 0.053425 & 0.121920 & 0.134732 & 0.082294 & 0.076375 & 0.166703 \\
\hline France & 0.002706 & 0.074290 & 0.054922 & 0.158203 & 0.171321 & 0.131431 \\
\hline Gabon & 0.000841 & 0.054425 & 0.050388 & 0.036104 & 0.080507 & 0.062812 \\
\hline Gambia & 0.024720 & 0.055433 & 0.113156 & 0.000000 & 0.030968 & 0.057536 \\
\hline Germany & 0.000695 & 0.064309 & 0.036048 & 0.141251 & 0.171543 & 0.090054 \\
\hline Guinea & 0.001682 & 0.084759 & 0.053483 & 0.054451 & 0.119605 & 0.102895 \\
\hline Guinea-Bissau & 0.001097 & 0.048020 & 0.000000 & 0.053217 & 0.006309 & 0.064040 \\
\hline Guyana & 0.000219 & 0.000098 & 0.018525 & 0.000000 & 0.059714 & 0.002256 \\
\hline India & 0.011007 & 0.143703 & 0.016520 & 0.145328 & 0.056914 & 0.136973 \\
\hline Indonesia & 0.169856 & 0.155472 & 0.281495 & 0.183793 & 0.071221 & 0.147292 \\
\hline Iran & 0.136763 & 0.117140 & 0.082906 & 0.058904 & 0.095568 & 0.123335 \\
\hline Iraq & 0.050244 & 0.129755 & 0.056622 & 0.068346 & 0.035544 & 0.127615 \\
\hline Italy & 0.001828 & 0.060082 & 0.064947 & 0.165875 & 0.135022 & 0.065931 \\
\hline
\end{tabular}

Volume 10 Issue 2, February 2021 www.ijsr.net

Licensed Under Creative Commons Attribution CC BY 
International Journal of Science and Research (IJSR)

ISSN: 2319-7064

SJIF (2019): 7.583

\begin{tabular}{|c|c|c|c|c|c|c|}
\hline Ivory Coast & 0.001499 & 0.048248 & 0.105441 & 0.050428 & 0.044607 & 0.068354 \\
\hline Jordan & 0.179510 & 0.195722 & 0.152473 & 0.120919 & 0.112363 & 0.193414 \\
\hline Kazakhstan & 0.044283 & 0.104721 & 0.062768 & 0.060567 & 0.134044 & 0.107441 \\
\hline Kenya & 0.055985 & 0.083003 & 0.031689 & 0.145811 & 0.060913 & 0.094401 \\
\hline Kuwait & 0.206680 & 0.136875 & 0.053876 & 0.065127 & 0.135066 & 0.114276 \\
\hline Kyrgyzstan & 0.018393 & 0.048800 & 0.136345 & 0.038143 & 0.071443 & 0.065732 \\
\hline Lebanon & 0.034300 & 0.061318 & 0.094282 & 0.058314 & 0.197090 & 0.089590 \\
\hline Libya & 0.018905 & 0.082353 & 0.083036 & 0.022156 & 0.033944 & 0.083086 \\
\hline Malaysia & 0.631998 & 0.261721 & 0.398836 & 0.175907 & 0.224503 & 0.317114 \\
\hline Mali & 0.000914 & 0.111158 & 0.086741 & 0.130951 & 0.018616 & 0.124530 \\
\hline Mauritania & 0.007058 & 0.083263 & 0.072662 & 0.132829 & 0.030168 & 0.092211 \\
\hline Morocco & 0.053791 & 0.134339 & 0.128020 & 0.135886 & 0.117561 & 0.138632 \\
\hline Mozambique & 0.000804 & 0.085344 & 0.108405 & 0.040986 & 0.029368 & 0.091348 \\
\hline Niger & 0.001572 & 0.073737 & 0.083298 & 0.087283 & 0.007242 & 0.084347 \\
\hline Nigeria & 0.095844 & 0.025294 & 0.091972 & 0.066146 & 0.046829 & 0.040216 \\
\hline Oman & 0.188579 & 0.203167 & 0.122484 & 0.135135 & 0.124625 & 0.143775 \\
\hline Pakistan & 0.193735 & 0.188114 & 0.065557 & 0.118612 & 0.041497 & 0.192850 \\
\hline Palestine & 0.081729 & 0.070844 & 0.078067 & 0.084600 & 0.102499 & 0.071340 \\
\hline Qatar & 0.199952 & 0.160576 & 0.118430 & 0.062176 & 0.279552 & 0.116400 \\
\hline Russia & 0.002267 & 0.068860 & 0.063596 & 0.093667 & 0.158037 & 0.067756 \\
\hline Saudi Arabia & 0.234106 & 0.157585 & 0.149814 & 0.086585 & 0.145596 & 0.154525 \\
\hline Senegal & 0.005449 & 0.129235 & 0.103000 & 0.111263 & 0.043674 & 0.144206 \\
\hline Sierra Leone & 0.001097 & 0.028708 & 0.008325 & 0.101231 & 0.019727 & 0.043136 \\
\hline Somalia & 0.012616 & 0.082060 & 0.000000 & 0.022156 & 0.003155 & 0.027640 \\
\hline South Africa & 0.089408 & 0.134372 & 0.040407 & 0.120168 & 0.097079 & 0.117396 \\
\hline Sri Lanka & 0.083740 & 0.083328 & 0.036222 & 0.148493 & 0.059447 & 0.089523 \\
\hline Sudan & 0.123854 & 0.178198 & 0.127540 & 0.041200 & 0.046918 & 0.068254 \\
\hline Suriname & 0.001463 & 0.045159 & 0.045812 & 0.023551 & 0.076064 & 0.048843 \\
\hline Syria & 0.024976 & 0.025749 & 0.037835 & 0.056919 & 0.065312 & 0.008030 \\
\hline Tajikistan & 0.015687 & 0.124391 & 0.021751 & 0.044205 & 0.035188 & 0.147425 \\
\hline Thailand & 0.016492 & 0.133006 & 0.183682 & 0.143826 & 0.092592 & 0.144538 \\
\hline Togo & 0.000402 & 0.047435 & 0.056491 & 0.069472 & 0.019371 & 0.076914 \\
\hline Tunisia & 0.036604 & 0.122635 & 0.177100 & 0.075749 & 0.087527 & 0.129673 \\
\hline Turkey & 0.075658 & 0.144548 & 0.309130 & 0.171293 & 0.110985 & 0.134816 \\
\hline Turkmenistan & 0.000256 & 0.084499 & 0.000000 & 0.000000 & 0.030123 & 0.088727 \\
\hline Uganda & 0.017955 & 0.110411 & 0.064511 & 0.105201 & 0.036655 & 0.118457 \\
\hline UnitedArabEmirates & 0.301792 & 0.294396 & 0.423158 & 0.568652 & 0.482507 & 0.344323 \\
\hline UnitedKingdom & 0.061799 & 0.110703 & 0.045986 & 0.104825 & 0.183450 & 0.109200 \\
\hline UnitedStates & 0.001316 & 0.097016 & 0.022971 & 0.073442 & 0.140442 & 0.077478 \\
\hline Uzbekistan & 0.002377 & 0.054457 & 0.004882 & 0.004775 & 0.079129 & 0.055479 \\
\hline Yemen & 0.024208 & 0.111646 & 0.011595 & 0.084439 & 0.041186 & 0.127914 \\
\hline
\end{tabular}

The weighted decision matrix is gotten by (7), the ideal solution and the negative ideal solution are obtained by (8) (9) and then, the ideal value and the negative ideal value are obtained by (10) (11). Results are shown in table 6 as follows.

Table 6: Ideal positive value and the ideal negative value

\begin{tabular}{|c|c|c|c|c|c|c|}
\hline & $\mathrm{C}_{1}$ & $\mathrm{C}_{2}$ & $\mathrm{C}_{3}$ & $\mathrm{C}_{4}$ & $\mathrm{C}_{5}$ & $\mathrm{C}_{6}$ \\
\hline $\mathrm{V}$ & 5.34400 & 5.77574 & 7.08768 & 1.03189 & 8.39364 & 6.76029 \\
+ & $\mathrm{E}-02$ & $\mathrm{E}-02$ & $\mathrm{E}-02$ & $\mathrm{E}-01$ & $\mathrm{E}-02$ & $\mathrm{E}-02$ \\
\hline $\mathrm{V}$ & 6.18412 & 1.91355 & 0.00000 & 0.00000 & 5.48755 & 4.42999 \\
- & $\mathrm{E}-06$ & $\mathrm{E}-05$ & $\mathrm{E}+00$ & $\mathrm{E}+00$ & $\mathrm{E}-04$ & $\mathrm{E}-04$ \\
\hline
\end{tabular}

The distance of every feasible solution from the ideal solution and the negative ideal solution is obtained according to (12) (13). The relative degree of approximation is determined according to (14). Criteria of seventy countries could be ranked by the relative degree of approximation and are shown in the table 7 .
Table 7: Distance, relative degree of approximation and rank position

\begin{tabular}{|c|c|c|c|c|}
\hline & $\mathrm{S}^{+}$ & $\mathrm{S}^{-}$ & & \\
\hline Afghanistan & 0.16203125 & 0.02885843 & 0.15117858 & 53 \\
\hline Albania & 0.148311243 & 0.03867603 & 0.20683778 & 36 \\
\hline Algeria & 0.148751761 & 0.04264154 & 0.22279537 & 34 \\
\hline Australia & 0.141082268 & 0.05352531 & 0.27504227 & 15 \\
\hline Azerbaijan & 0.138821225 & 0.05599891 & 0.28743903 & 14 \\
\hline Bahrain & 0.125976442 & 0.06356507 & 0.33536226 & 8 \\
\hline Bangladesh & 0.142892005 & 0.04489148 & 0.23905977 & 25 \\
\hline Benin & 0.16083063 & 0.02864424 & 0.151177 & 54 \\
\hline Brazil & 0.155731643 & 0.04578908 & 0.22721771 & 30 \\
\hline Brunei & 0.130877381 & 0.06471582 & 0.33086946 & 9 \\
\hline Burkina Faso & 0.152456955 & 0.03826546 & 0.20063431 & 42 \\
\hline Cameroon & 0.153662764 & 0.03260063 & 0.17502435 & 49 \\
\hline Canada & 0.145300654 & 0.04251118 & 0.22634984 & 31 \\
\hline Chad & 0.162841315 & 0.02391938 & 0.128075 & 60 \\
\hline China & 0.143384393 & 0.04510078 & 0.23928026 & 24 \\
\hline Djibouti & 0.159635288 & 0.02550701 & 0.13776978 & 57 \\
\hline Egypt & 0.140957519 & 0.0502844 & 0.26293609 & 17 \\
\hline France & 0.136840458 & 0.05118313 & 0.27221654 & 16 \\
\hline Gabon & 0.162010086 & 0.02345598 & 0.12647045 & 62 \\
\hline Gambia & 0.165889356 & 0.02495247 & 0.13074948 & 59 \\
\hline Germany & 0.143265445 & 0.04480353 & 0.23822926 & 26 \\
\hline Guinea & 0.152659339 & 0.03541988 & 0.18832427 & 47 \\
\hline
\end{tabular}

Volume 10 Issue 2, February 2021 www.ijsr.net

Licensed Under Creative Commons Attribution CC BY 


\section{International Journal of Science and Research (IJSR) \\ ISSN: 2319-7064 \\ SJIF (2019): 7.583}

\begin{tabular}{|c|c|c|c|c|}
\hline -Bissau & 0.169837308 & 0.01814131 & 0.09650732 & 67 \\
\hline Guyana & 0.177055902 & 0.01031663 & 0.05505948 & 70 \\
\hline India & 0.145978719 & 0.04779946 & 0.24667103 & 21 \\
\hline Indonesia & 0.119689851 & 0.07363245 & 0.38087924 & 4 \\
\hline Iran & 0.145535335 & 0.04232622 & 0.22530538 & 32 \\
\hline Iraq & 0.152742985 & 0.03932355 & 0.20473919 & 40 \\
\hline Italy & 0.14330363 & 0.04295797 & 0.23063246 & 29 \\
\hline Ivory Coas & 0.159963224 & 0.02655751 & 0.1423837 & 56 \\
\hline Jordan & 0.12457631 & 0.06787027 & 0.35267069 & 5 \\
\hline Kazakhstan & 0.147771319 & 0.04014414 & 0.21362873 & 35 \\
\hline Kenya & 4938 & 0.0 & 7203 & 41 \\
\hline & 0827 & 0.0 & 0156 & 20 \\
\hline Kyrgyzstan & 0.157143917 & 0.030977 & 0.16466536 & 51 \\
\hline Lebanon & 145678863 & 1544 & 0.23243817 & 27 \\
\hline bya & 8643 & 0.0 & 2207 & 55 \\
\hline & & & 0.6 & 2 \\
\hline ali & 236 & & 206 & 33 \\
\hline Mauritania & & 6468 & 34276 & 44 \\
\hline Morocco & 8167 & & 82661 & 13 \\
\hline $\mathrm{Moz}$ & 511 & 768 & & 50 \\
\hline ser & & & 0.1 & 52 \\
\hline & & 0.0 & & 58 \\
\hline & & & 5247 & 7 \\
\hline $\mathrm{Pa}$ & & & & 11 \\
\hline $\mathrm{Pa}$ & 4451 & 968 & 2478 & 48 \\
\hline & & & 0.3 & 6 \\
\hline & & & & 38 \\
\hline Saud & & & 253 & 10 \\
\hline & & & 077 & 22 \\
\hline Sierr & 1011 & 0.0 & 58386 & 64 \\
\hline & & & & 69 \\
\hline Sou & 498 & & 0.2 & 23 \\
\hline Sri Lanka & & & 571 & 37 \\
\hline Idan & 6503 & & 3872 & 28 \\
\hline Suriname & 0906 & 591 & 1976 & 65 \\
\hline & 5879 & 986 & & 68 \\
\hline Tajikistal & 2144 & 0.0 & 0.19 & 43 \\
\hline & & & & 12 \\
\hline Togo & 5127 & 1193 & 83193 & 61 \\
\hline nisia & 7061 & 594 & 5965 & 18 \\
\hline & & 891 & 9432 & 3 \\
\hline Turkmenistan & 4844 & 7489 & 43981 & 63 \\
\hline Uganda & 0.150539599 & 0.03881131 & 0.20497029 & 39 \\
\hline UnitedArabEmirates & 0.027921285 & 0.17640899 & 0.86335219 & 1 \\
\hline United & 0.1397715 & 0.04841333 & 0.2572648 & 19 \\
\hline & & & 0.19391514 & 45 \\
\hline Uzbekistan & & 0.01997663 & 0.10569689 & 66 \\
\hline Yemen & 0.155236887 & 0.03706877 & 0.19275964 & 46 \\
\hline
\end{tabular}

changes, but their rank is more precise thanks to the objectivity of the method used in this research.

Table 8: Ranking comparation (EWM+TOPSIS vs GIER) for top 10 countries

\begin{tabular}{|c|c|c|}
\hline \multicolumn{1}{|c|}{} & EWM+TOPSIS & GIER \\
\hline United Arab Emirates & 1 & 2 \\
\hline Malaysia & 2 & 1 \\
\hline Turkey & 3 & 13 \\
\hline Indonesia & 4 & 5 \\
\hline Jordan & 5 & 7 \\
\hline Qatar & 6 & 10 \\
\hline Oman & 7 & 6 \\
\hline Bahrain & 8 & 3 \\
\hline Brunei & 9 & 11 \\
\hline Saudi Arabia & 10 & 4 \\
\hline
\end{tabular}

\section{Discussion}

When working with multicriteria decision making methods, determining the weight of criteria is really important. In this research, Entropy Weight Method appears as an effective solution to get objective results and to avoid subjectivity when calculating the weights of the criteria.

In this research, seventy countries have been evaluated according to theEWM + TOPSIS method. The study is feasible and rational, so it can be applied to identify the advances of any country when facing the Halal standards and therefore, the methodology should be applied widely for evaluation matters in other relevant fields.

Compared to equally ranked methodologies, this combination of Entropy Weight and TOPSIS is clear, reasonable and it provides more accurate results because the criteria are weighed based on objective information and it is reasonable to think that not all criteria are equally important to determine the improvements of a country in terms of Halal principles.

\section{References}

[1] Alimovich FE,Habibulloevna KS and Bahodirovna DN. Central features of Halal Tourism and halal food. Academy. 2020; 3-54.

[2] Thomson Reuters. State of the global Islamic economy report 2019/20. 2019.

\subsection{Evaluation results analysis and comparison}

As was anticipated before, results provided by the EWM + TOPSIS are slightly different to those provided by the latest Global Islamic Economy Report (GIER). In table 8. positions of the top 10 countries analyzed in the present research are compared with those in the GIER.

UAE and Malaysia are keeping the first positions but with different order. Comparing the GIER 2018 with the 2019 version, the case of Turkey is striking. In the GIER the country experiences an important risefrom the previous year, up to position number 13. But the EWM + TOPSIS method gives it the third position. Also Bahrain can be considered a case study because the GIER places it in the third position but theEWM + TOPSIS analysisplaces it only ineighth place. The rest of the countries do not present significant

3] Yoon K and Hwang CL. TOPSIS (technique for order preference by similarity to ideal solution) - a multiple attribute decision making, w: Multiple attribute decision making - methods and applications, a state-of-the-at survey. Berlin: Springer Verlag. 1981; 128-140.

[4] Thomson Reuters. State of the global Islamic economy report 2013. 2013.

[5] Saaty TL. A scaling method for priorities in hierarchical structures, Journal of mathematical psychology. Elsevier.1977. vol 15- 3. pages 234 - 281. 1977.

[6] E Bottani E and Rizzi A. A fuzzy TOPSIS methodology to support outsourcing of logistics services. Supply Chain Management: An International Journal, Emerald Group Publishing Limited. 2006

[7] Ashrafzadeh M,RafieiFM, Isfahani NM andZare Z. Application of fuzzy TOPSIS method for the selection

\section{Volume 10 Issue 2, February 2021 www.ijsr.net}


of Warehouse Location: A Case Study. Interdisciplinary Journal of Contemporary Research in Business. 2012; Vol. 3 - 9. pages 655-671.

Volume 10 Issue 2, February 2021 www.ijsr.net 